\title{
Protostellar mergers in protoclusters and the origin of ultra-luminous X-ray sources
}

\author{
Roberto Soria ${ }^{1}$ \\ ${ }^{1}$ Harvard-Smithsonian Center for Astrophysics, 60 Garden st, Cambridge, MA 02138, USA \\ email: rsoria@cfa.harvard.edu
}

\begin{abstract}
I suggest that stellar coalescence in mid-size protoclusters $\left(M \sim 10^{3.5}-10^{4.5} M_{\odot}\right)$ is a possible scenario for the formation of ultraluminous X-ray sources (ULXs). More massive superstar-clusters are not needed, since the most likely ULX mass range is only $\sim 30-200 M_{\odot}$; in fact, they are very rarely found at or very near ULX positions. Protostellar envelopes and gas accretion favour captures and mergers in dense cores of embedded clusters. Moreover, protoclusters with masses $\sim 10^{3.5}-10^{4.5} M_{\odot}$ are likely to disperse quickly into loose OB associations, where most ULXs are found. Sufficiently high protostellar density may be achieved when clustered star formation is triggered by galaxy collisions and mergers. Low metallicity may then be necessary to ensure that a large fraction of the stellar mass ends up in a black hole. In this scenario, most ULXs are naturally explained as the extreme end of the high-mass X-ray binary population.
\end{abstract}

Keywords. black hole physics, stars: formation, galaxies: star clusters, X-ray: binaries.

\section{Introduction: young or old black holes in ULXs?}

Ultraluminous X-ray sources (ULXs) are accreting compact objects with an apparent X-ray luminosity $L_{\mathrm{x}}>10^{39} \mathrm{erg} \mathrm{s}^{-1}$, i.e., higher than the Eddington limit of a Galactic stellar-mass black hole (BH). Their nature is still hotly debated. Three fundamental, unsolved questions are: a) are these sources beamed towards us, or truly ultra-luminous? b) in the latter case, are they emitting above their classical Eddington limit, or are they more massive than stellar-mass BHs (i.e., $\left.M \gtrsim 30 M_{\odot}\right)$ ? c) if the accreting BHs are indeed more massive than "typical" stellar remnants, were they formed in recent star-formation processes, or are they old relics from the early Universe?

I shall not review here the different arguments in favour or against the alternative scenarios in questions $a$ ) and b) (see Miller \& Colbert 2004 for a review). I shall instead assume for the sake of this discussion that most ULXs are not significantly beamed sources, and that they do not significantly violate the Eddington limit. Hence, I shall accept that ULXs in nearby galaxies are powered by accreting BHs more massive than those found in our Galaxy ("intermediate-mass BHs", IMBHs, with masses $M \gtrsim 30 M_{\odot}$ ). As for the third argument, it has become clear that most ULXs (in particular, those brighter than $\approx 3 \times 10^{39} \mathrm{erg} \mathrm{s}^{-1}$ ) are found in star-forming galaxies, not in ellipticals (Irwin et al. 2004; Swartz et al. 2004). Colliding or merging galaxies (e.g., the Antennae) contain a large number of ULXs, often associated with starburst regions, and typical stellar populations around ULXs tend to be young $(\sim 10-50 \mathrm{Myr})$. The X-ray luminosity function of accreting sources above $10^{39} \mathrm{erg} \mathrm{s}^{-1}$ is also consistent with ULXs being the bright end of the high-mass X-ray binary distribution, normalized to the star formation rate (Gilfanov et al. 2004). This is not absolute proof that the accreting BHs are themselves young: for example, they could be old Population-III remnants that have recently captured a young donor star while crossing a dense star-forming region. However, the simplest scenario we need to investigate is that both the $\mathrm{BH}$ progenitors and the donor stars are co-eval. 


\section{The super-star-cluster scenario and its shortcomings}

Runaway stellar mergers of main-sequence $\mathrm{O}$ stars in the collapsed core of a young super-star-cluster $\left(M \sim 10^{6} M_{\odot}\right.$, size $\left.\sim 1 \mathrm{pc}\right)$ have been proposed as a viable mechanism to produce a stellar object with a mass up to $\sim 1000 M_{\odot}$ at the cluster center, which would then collapse into an IMBH (Portegies Zwart \& McMillan 2002; Gürkan et al. 2004). This process has been used to explain, for example, the brightest ULX in the irregular starburst galaxy M 82 (Portegies Zwart et al. 2004). However, this scenario cannot be used to explain most other ULXs. In fact, very few of them are inside a massive, compact star cluster. In a few cases (e.g., the Antennae), there are super-star-clusters nearby, but the X-ray source is displaced by $\sim 100-300$ pc. In most other cases, there are no superstar-clusters, just OB associations, or a group of a few OB stars near the ULX position.

If ULXs were born in compact, massive clusters, what happened to them? Was the BH expelled from the cluster (unlikely, if it is an IMBH), or, more likely, has the parent cluster already dissolved? Dispersion of most young star cluster into expanding OB associations is seen in the Antennae, on timescales $\sim 10^{7}$ yr (Fall et al. 2005). I suggest that the runaway merger scenario ought to take into account the following two basic observational constraints:

- the X-ray luminosity distribution has a cut-off at $\approx 3 \times 10^{40} \mathrm{erg} \mathrm{s}^{-1}$ (Gilfanov et al. $2004)$, with only very few sources brighter than that. This suggests that the required mass range for the accreting IMBHs is only $\sim 30-200 M_{\odot}$. More massive IMBHs $\left(M \sim 10^{3} M_{\odot}\right)$ have been invoked (Miller et al. 2004) based on the detection of X-ray spectral features interpreted as BH mass indicators. In my opinion, those arguments are not convincing (see, e.g., Gierliński \& Done 2004 for an alternative explanation of the thermal component at $k T \approx 0.15 \mathrm{keV}$ ) and there is no reason to invoke such massive IMBHs.

- most ULXs are located in OB associations, with sizes $\sim 100 \mathrm{pc}$ and $M \sim 10^{3.5}$ $10^{4.5} M_{\odot}$, rather than inside compact clusters with sizes $\lesssim$ a few pc and $M \sim 10^{5}-10^{6} M_{\odot}$.

\section{A protocluster scenario}

I propose the following two ingredients to reconcile the merger scenario with the two observational constraints mentioned in Section 2:

\subsection{Protostellar mergers inside an embedded cluster}

In the super-star-cluster scenario, the timescale available for stellar mergers is $\lesssim 3 \mathrm{Myr}$ (lifetime of a main-sequence $\mathrm{O}$ star). If we require the collisions to occur already in the protocluster stage, the time available is only $\lesssim 0.3 \mathrm{Myr}$. Such timescales may still be long enough to allow runaway core collapse of mid-size clusters, with masses $\lesssim 10^{5} M_{\odot}$,

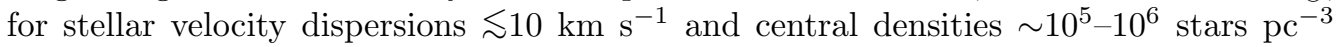
(Soria 2005). More significantly, the coalescence rates are increased by a few orders of magnitude when they involve interactions between protostars, surrounded by envelopes or disks, with radii up to a few hundred AU, i.e., $\gtrsim 1 / 10$ of typical separations between protostars in a cluster core (Bally \& Zinnecker 2005; Elmegreen \& Shadmehri 2003). In disk- or envelope-assisted interactions, angular momentum of the interacting stars can be efficiently dissipated by viscous processes and envelope/disk ejection. This favours the formation of massive binary protostars. Subsequent orbital decay and final coalescence are also strongly enhanced during the embedded cluster phase: gas accretion onto the protostars leads to orbital shrinking and further dissipative interactions with the circumstellar material (Bally \& Zinnecker 2005; Bonnell \& Bate 2002). 


\subsection{Mid-size protoclusters, not super-star-clusters}

Studying the possible formation of a $10^{3} M_{\odot}$ IMBH in a super-star-cluster may give us clues on globular cluster evolution, but may not be relevant to the observed ULX population, which is generally located in smaller, unbound OB associations. However, protoclusters in the mass range $\sim 10^{3.5}-10^{4.5} M_{\odot}$, with central densities $\sim 10^{6}$ protostars $\mathrm{pc}^{-3}$ may be more suitable. We speculate that these protoclusters can be dense enough to allow stellar coalescence up to the required IMBH progenitor masses (perhaps $\sim 100$ $\left.400 M_{\odot}\right)$. At the same time, they are small enough that they tend not to survive the embedded phase, evolving into unbound OB associations. There are two main reasons why protoclusters in this mass range may be less likely to survive into a bound cluster (Kroupa \& Boily 2002; although, Fall et al. 2005 argue instead that the survival rate within the first $\sim 10^{7} \mathrm{yr}$ is mass-independent):

- they are massive enough to contain many O stars, which ionize all the cluster gas; but at the same time, they are not massive enough to retain the ionized gas $\left(k T_{\text {gas }} \sim 10^{4} \mathrm{~K}\right.$ corresponds to $c_{\mathrm{S}} \sim 10 \mathrm{~km} \mathrm{~s}^{-1}$, larger than the escape velocity from the cluster); hence, they may evaporate "explosively" (Kroupa \& Boily 2002);

- if an IMBH progenitor is produced via stellar coalescence in the protocluster core, for example by the final merging of two 100- $M_{\odot}$ stars, the gravitational energy released by the merger ( $\gtrsim 10^{51}$ erg: Bally \& Zinnecker 2005) may be larger than the binding energy of the protocluster.

Thus, I speculate that if stellar coalescence occurs in mid-size protoclusters (rather than super-star-clusters), it will be easier to explain a population of IMBHs with masses $\sim 30$ $200 M_{\odot}$ observed in OB associations (leftover of the dispersed parent protoclusters). An additional advantage of this scenario is that the formation of ULX progenitors would be essentially the same physical process as the formation of the progenitors of BH high-mass X-ray binaries such as Cyg X-1. This class of massive binary systems are also thought to originate from the coalescence of less massive protostars inside embedded clusters (Bally \& Zinnecker 2005). This would be consistent with the observational finding that ULXs may simply be the upper end of the high-mass X-ray binary distribution.

\section{Open problems: from massive star to massive $\mathbf{B H}$}

Whatever the stellar coalescence scenario (super-star-clusters or mid-size protoclusters), forming a very massive star in the cluster core is not enough to have a ULX yet: first, the star has to collapse into a sufficiently massive $\mathrm{BH}$. The final mass of an $\mathrm{O}$ star before core collapse is generally much less than the initial mass, due to stellar wind losses. For example, at solar metallicities, a $120 M_{\odot}$ star explodes as a supernova with a core mass only $\approx 20 M_{\odot}$ (e.g., Vanbeveren 2004 ). Low metallicity reduces this problem, allowing for the formation of more massive remnants, for two reasons. Firstly, mass loss in the stellar wind is much reduced: $\dot{M} \sim Z^{0.86}$ for $10^{-2} \lesssim Z / Z_{\odot} \lesssim 1$ (Vink \& de Koter 2005). Secondly, at sub-solar (but not primordial) metallicity, all stars with masses $\gtrsim 40 M_{\odot}$ are thought to collapse directly into a BH (Heger et al. 2003). Weaker winds and direct BH collapse of the progenitor may be the reason why ULXs appear to prefer low-metallicity environments, as originally suggested by Pakull \& Mirioni (2002).

An additional requirement for ULX formation is that the BH has a Roche-lobe-filling companion star able to transfer $\gtrsim 10^{-6} M_{\odot} \mathrm{yr}^{-1}$. This is a comparatively minor problem: such steady mass transfer rates are possible for donor stars $\gtrsim 10 M_{\odot}$, over their nuclear timescale (a few $10^{6} \mathrm{yr}$ ) (Rappaport et al. 2005). Direct BH collapse of the primary may increase the likelihood of retaining a companion star, which will later become the donor. 
As an aside, I suggest that future optical measurements of their proper motion distribution may reveal whether ULXs are powered by beamed or super-Eddington stellar-mass $\mathrm{BHs}$ (in which case they would tend to have higher velocities, from SN kicks), or are more massive systems, formed via direct BH collapse (in which case no kick is expected).

\section{Conclusions}

I argue that one can explain the vast majority of ULXs with accreting BHs with masses $\sim 30-200 M_{\odot}$. Higher masses are probably not required. Thus, we do not need to invoke runaway merger processes in super-star-clusters $\left(M \sim 10^{6} M_{\odot}\right)$, which are not generally found at or near ULX locations. Coalescence of a few massive stars in a mid-size cluster $\left(\sim 10^{3.5}-10^{4.5} M_{\odot}\right)$ may be enough to explain the BH progenitors.

Moreover, I suggest that the merger process should occur in the protocluster stage: it is much easier to capture and merge protostars (surrounded by large disks or envelopes) than main-sequence stars, for the same stellar density and velocity dispersion. Core densities $\sim 10^{6}$ (proto)stars $\mathrm{pc}^{-3}$ are required for mergers to become significant. Such high densities are probably achieved when clustered star formation is triggered by molecular cloud collisions in merging galaxies (e.g., Keto et al. 2005). This may explain the preferential association of ULXs with tidally-disturbed environments.

Protoclusters in the $10^{3.5}-10^{4.5} M_{\odot}$ mass range are known to disperse quickly, evolving into OB associations rather than bound clusters. This is in agreement with the fact that most ULXs are found in mid-size stellar groups or OB associations rather than massive, bound clusters. Low metal abundance may be the other additional ingredient, ensuring low mass-loss in the stellar wind of the progenitor, followed by its direct BH collapse. In summary, perhaps the most important keys to understand ULX formation will come from infrared, sub-mm and radio studies of massive star formation in embedded clusters.

\section{References}

Bally, J. \& Zinnecker, H. 2005, ApJ 129, 2281

Bonnell, I.A. \& Bate, M.R. 2002, MNRAS 336, 659

Elmegreen, B.G. \& Shadmehri, M. 2003, MNRAS 338, 817

Fall, S.M., Chandar, R., \& Whitmore, B.C. 2005, ApJ (Letters), in press, astro-ph/0509293

Gierliński, M. \& Done, C. 2004, MNRAS (Letters) 349, 7

Gilfanov, M., Grimm, H.-J., \& Sunyaev, R. 2004, Nucl. Phys. B Proc. Suppl. 132, 369

Gürkan, M.A., Freitag, M., \& Rasio, F.A. 2004, ApJ 604, 632

Heger, A., Fryer, C.L., Woosley, S.E., Langer, N., \& Hartmann, D.H. 2003, ApJ 591, 288

Irwin, J.A., Bregman, J.N., \& Athey, A.E. 2004, ApJ (Letters) 601, L143

Keto, E., Ho, L.C., \& Lo, K.-Y. 2005, ApJ submitted, astro-ph/0508519

Kroupa, P. \& Boily, C.M. 2002, MNRAS 336, 1188

Miller, M.C. \& Colbert, E.J.M. 2004, Int. J. Mod. Phys. D 13, 1, astro-ph/0308402

Miller, J.M., Fabian, A.C., \& Miller, M.C. 2004, ApJ (Letters) 614, 117

Pakull, M.W. \& Mirioni, L. 2002, in the Proc. of New Visions of the X-ray Universe in the XMM-Newton and Chandra Era, astro-ph/0202488

Portegies Zwart, S.F., et al. 2004, Nature 428, 724

Portegies Zwart, S.F. \& McMillan, S.L.W. 2002, ApJ 576, 899

Rappaport, S.A., Podsiadlowski, Ph., \& Pfahl, E. 2005, MNRAS 356, 401

Soria, R. 2005, Proc. XXII Texas Symposium on Relativistic Astrophysics, astro-ph/0503340

Swartz, D.A., Ghosh, K.K., Tennant, A.F., \& Wu, K. 2004, ApJS 154, 519

Vanbeveren, D. 2004, in the Proc. of Evolution of Massive Stars, Mass Loss and Winds, p. 141, astro-ph/0302199

Vink, J.S. \& de Koter, A. 2005, A\&A in press, astro-ph/0507352 


\section{Discussion}

MirABEL: Why there are no IMBHs found in the MW and MCs?

SORIA: If we refer to young, accreting ULXs, perhaps simply because of statistics. The rate of clustered star formation in low-metallicity environments is relatively low for MW and MCs. After all, we only see a dozen bright ULXs $\left(\mathrm{L}_{x}>10^{40} \mathrm{erg} / \mathrm{s}\right)$ within $\sim 5 \mathrm{Mpc}$, so the probability of formation must be low.

LIPUNOV: It seems that some of the ULXs can be NS, for example, well-known X-ray pulsar AO538-66 sometimes has luminosity up to $10^{39} \mathrm{erg} / \mathrm{s}$. As shown in Lipunov (1982) all matter comes to magnetosphere can come to surface. In this case luminosity can much larger than Eddington one. The spectra is not usual.

SORIA: But spectra of ULXs are very different from X-ray pulsars. [Answer recorded by former person.] 


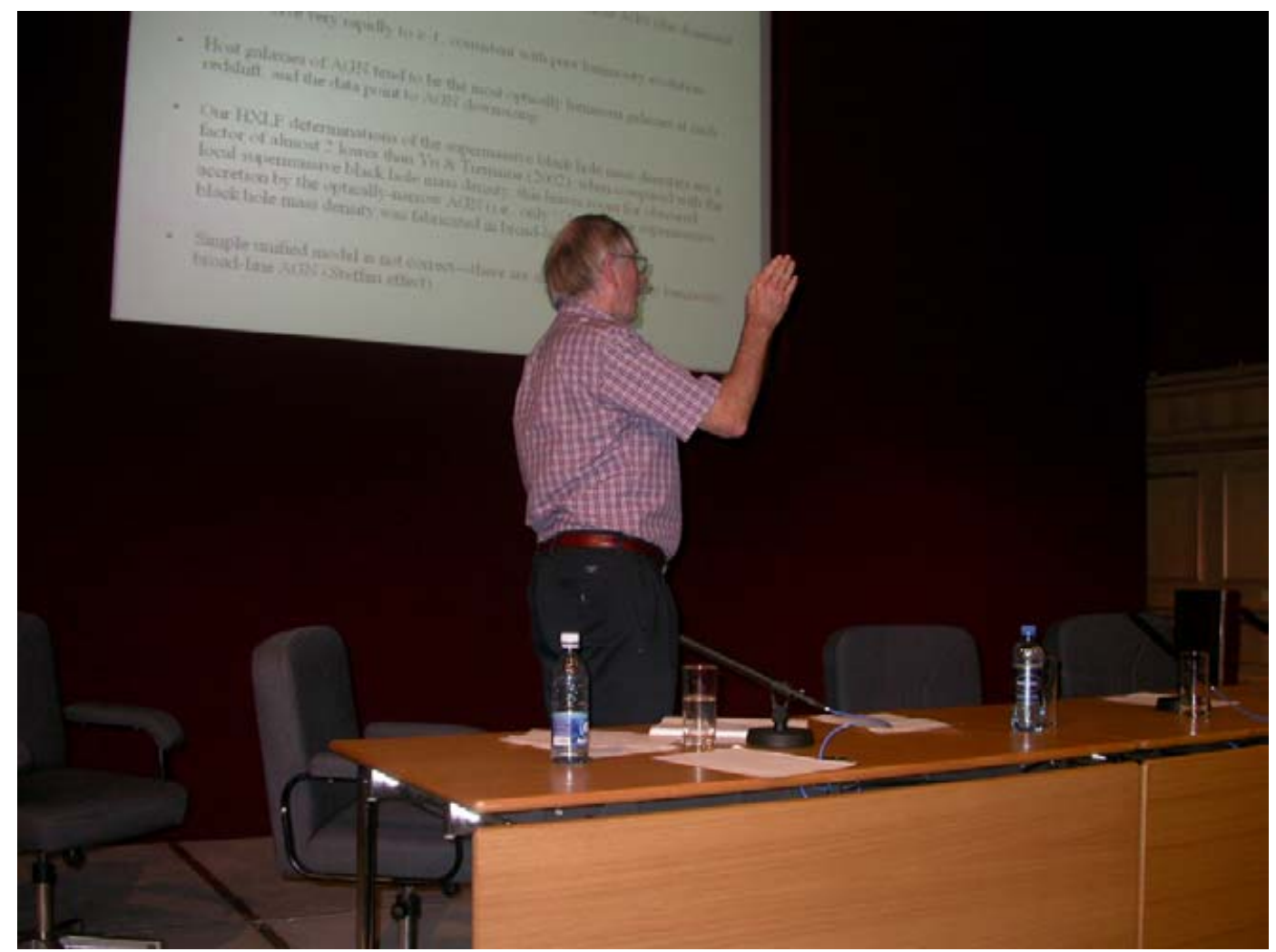

George Miley keeping time strictly. 